\title{
Recurrent and Persistent Pneumonia in Dhaka Shishu (Children) Hospital: Clinical Profile and Etiology
}

\author{
NAZIA HOSSAIN ${ }^{1}$, KAMRUZZAMAN KAMRUL ${ }^{2}$, AKHAND TANZIH SULTANA ${ }^{3}$, MD.SHAKIBUR RAHMAN ${ }^{4}$, \\ MD RUHUL AMIN 5
}

\begin{abstract}
:
Background: Recurrent and persistent pneumonia have become a challenge for the physicians. The aim of this study was to evaluate the clinical profile and determine the underlying causes of recurrent and persistent pneumonia among the children admitted in Dhaka Shishu (Children) Hospital.

Methods: In this study, retrospective analysis of medical records of the children was done, who met the criteria of recurrent pneumonia and persistent pneumonia. Analyzed Patients were admitted in Dhaka Shishu (Children) Hospital from January 2016 to Decembe 2016.

Results: A total of 30 patients were analyzed during the study period. Twenty-four patients presented with recurrent pneumonia (80\%) and six patients presented with persistent pneumonia (20\%) during the study a period. After analysis of clinical profile, identified causes were pulmonary TB (23.3\%), congenital heart disease (13.3\%), cystic fibrosis (16.6\%), aspiration syndrome (13.3\%), bronchial asthma (10\%), immune deficiency disorders (10\%), bronchiectasis (6.6\%), congenital lung agenesis (3.3\%), unknown cause (3.3\%).

Conclusion: The most frequent underlying cause for recurrent and/or persistent pneumonia was pulmonary TB followed by congenital heart disease and cystic fibrosis among the patients admitted in Dhaka Shishu (Children) Hospital.
\end{abstract}

Keywords: Persistent pneumonia, Recurrent Pneumonia, Cystic Fibrosis, Pulmonary TB.

\section{Introduction:}

Pneumonia is one of the major causes of under-five mortality in Bangladesh. Recurrent pneumonia and persistent pneumonia has become a challenge for our children. A small portion of pneumonia becomes recurrent and persistent ${ }^{1-2}$. Recurrent pneumonia is defined as at least 2 episodes of pneumonia in a 1-year period or more than 3 episodes of pneumonia at any time with

1. Resident Medical Officer, Dept. of Pediatric Pulmonology, Dhaka Shishu (Children) Hospital

2. Assistant Professor, Dept. of Pediatric Pulmonology, Dhaka Shishu (Children) Hospital

3. Assistant Professor, Dept. of Pediatric Pulmonology, Dhaka Shishu(Children) Hospital

4. FCPS Part-II student (Pediatric Pulmonology), Dhaka Shishu (Children) Hospital

5. Professor and head, Dept. of Pediatric Pulmonology, Dhaka Shishu (Children) Hospital

Correspondence: Dr. Nazia Hossain, Resident Medical Officer, Dept. of Pediatric Pulmonology, Dhaka Shishu (Children) Hospital Contact: 01816317167, Email: naziahossain7@yahoo.com

Received: 14 January 2018
Accepted: 25 October 2018 radiographic clearance between episodes ${ }^{3}$. By this definition, recurrent pneumonia occurs in $6.4 \%-$ $9 \%$ of all children with pneumonia ${ }^{4-7}$. Persistent pneumonia is defined as features of lower respiratory tract infection (i.e. cough, tachypnea and fever with or without chest retractions) with radiological evidence of infiltrates or consolidation in the lungs persisting for 30 days or more, despite receiving antibiotics for a minimum period of 10 days. ${ }^{8,9}$. There are limited data on the factors predisposing to recurrence of pneumonia or persistence of pneumonia ${ }^{4,10-11}$. The purpose of this study is to describe the predisposing causes of recurrent pneumonia and persistent pneumonia.

\section{Materials and methods:}

This is a retrospective study, conducted in the department of Pediatric Respiratory Medicine (Pulmonology) unit of Dhaka Shishu (Children) Hospital, Bangladesh. Our study was conducted from 
January 2016 to December 2016. We retrospectively analyzed the cases, aged between 2 months to 12 years, admitted and treated in our department during the study period. Patients were admitted directly or referred from other units, clinics or hospitals. Patients were included according to the definition of recurrent and persistent pneumonia. Radiographic evidence of recurrent pneumonia and persistent pneumonia was the criteria for inclusion. However, patients with critical conditions like severe respiratory insufficiency were excluded from the study. Diagnosis was made with history, clinical features and relevant investigations. Data for all patients regarding demographic profile, episodes of pneumonia and relevant investigations including Chest $X$-ray, CT scan of chest were collected. Data obtained from the registrar of pediatric pulmonology unit. Collected data were analyzed by SPSS version 16 .

\section{Results:}

During the one-year period (January, 2016 to December 2016) we analyzed 30 patients who satisfied the criteria of either recurrent or persistent pneumonia.

Their age ranged from 2 months to 12 years. The mean age of presentation was $3.0 \pm 2.8$ years. Four children (13.3\%) had onset of symptoms before 6 months of age, 10 (33.3\%) between 6 months to 2 years, $13(43.3 \%)$ between 2 to 5 years and $3(10.0 \%)$ after the age of 5 years.

Patients were $18(60 \%)$ males and $12(40 \%)$ females.

Among the presenting symptoms cough was seen in all cases $(100 \%)$, then breathlessness, fever, wheeze and failure to thrive were documented. Physical examination revealed evidence of tachypnea, clubbing, chest deformity, abnormal sound on auscultation, rickets and malnutrition (moderate/Severe) (Table 2). Most of the children were found malnourished $(80 \%)$.

The chest radiograph showed evidence of pneumonia in all cases. CT scan of chest was performed in 17 cases which revealed bronchiectasis in $3(10 \%)$ cases, collapse in $4(13.3 \%)$ cases, pneumonia in $8(26.6 \%)$ cases, agenesis of lung in $1(3.3 \%)$ cases, congenital lobar emphysema in $1(3.3 \%)$ cases.
We had 24 patients with recurrent pneumonia $(80 \%)$ and 6 patients with persistent pneumonia (20\%). Among the etiological factors Gastro-esophageal reflux disease(GERD) were found in $2(6.6 \%)$ patients who were diagnosed by clinical suspicion and using barium swallow, esophageal pH manometry. 2(6.6\%) patients had definite history of foreign body (FB) aspiration. This history was confirmed by bronchoscopic removal of foreign body. Three patients $(10 \%)$ had immune deficiency disorders. Congenital heart disease was confirmed by echocardiography in 5 patients (16.6\%).Among these patients, 2 patients were ventricular septal defect (VSD), 1 had Patent Ductus arteriosus (PDA), 1 with transposition of great arteries (TGAs), and one with complex congenital heart disease. Three patients $(10 \%)$ had bronchial asthma. Cystic fibrosis was diagnosed in $4(13.3 \%)$ patients. Pulmonary TB was diagnosed in $7(23.3 \%)$ patients; one patient $(3.3 \%)$ had congenital anomalies of the respiratory tract that was congenital agenesis of right lung. Two (6.6\%) patients had bronchiectasis. In 1 child, no underlying cause could be identified.

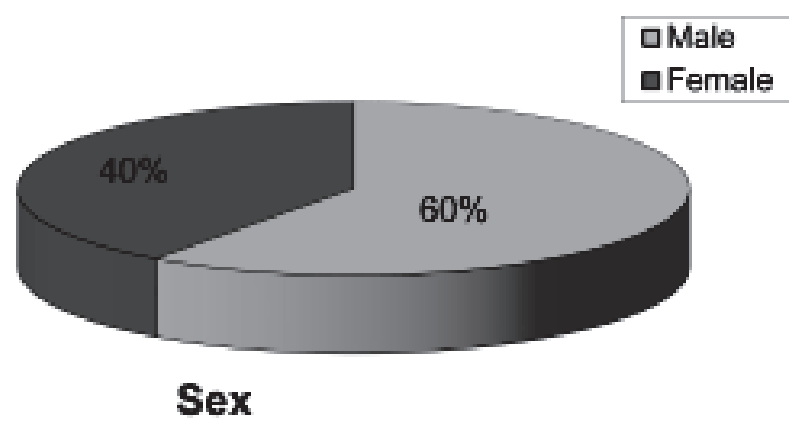

Fig.-1: Pie chart showing the sex distribution of the studied patients.

Table-I

Types of Pneumonia $(n=30)$

\begin{tabular}{lcc}
\hline Patients No (\%) & $\begin{array}{c}\text { Recurrent } \\
\text { Pneumonia }\end{array}$ & $\begin{array}{c}\text { Persistent } \\
\text { Pneumonia }\end{array}$ \\
\hline Number & 24 & 6 \\
Percentage & $80 \%$ & $20 \%$ \\
\hline
\end{tabular}


Table II

Clinical Profile and radiologic Profile $(n=30)$

\begin{tabular}{|c|c|c|}
\hline Characteristics & Number & Percentage \\
\hline $\begin{array}{ll}\text { Presenting feature } \\
\text { - } & \text { Cough } \\
\text { - } & \text { Breathlessness } \\
\text { - } & \text { Fever } \\
\text { - } & \text { Wheeze } \\
\text { - } & \text { Failure to Thrive }\end{array}$ & $\begin{array}{l}30 \\
19 \\
26 \\
14 \\
17\end{array}$ & $\begin{array}{l}100 \% \\
63.3 \% \\
86.6 \% \\
46.6 \% \\
56.6 \%\end{array}$ \\
\hline 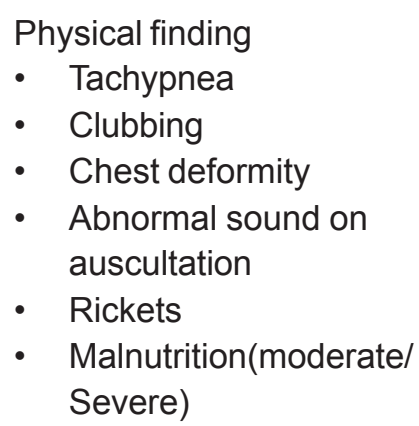 & $\begin{array}{c}21 \\
6 \\
12 \\
26\end{array}$ & $\begin{array}{c}70 \% \\
20 \% \\
40 \% \\
86.6 \% \\
3.3 \% \\
80 \%\end{array}$ \\
\hline $\begin{array}{l}\text { Chest Radiograph } \\
\text { - } \text { Collapse } \\
\text { - } \text { Consolidation } \\
\text { - } \text { Bronchopneumonia } \\
\text { - } \text { Bronchiectasis } \\
\text { - Hyperinflation } \\
\text { - Agenesis of lung }\end{array}$ & $\begin{array}{l}5 \\
7 \\
8 \\
5 \\
4 \\
1\end{array}$ & $\begin{array}{c}16.6 \% \\
23.3 \% \\
26.6 \% \\
16.6 \% \\
13.3 \% \\
3.3 \%\end{array}$ \\
\hline 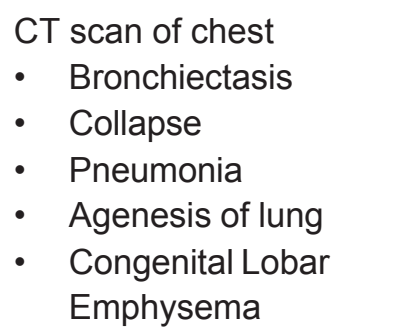 & $\begin{array}{l}3 \\
4 \\
8 \\
1 \\
1\end{array}$ & $\begin{array}{c}10 \% \\
13.3 \% \\
26.6 \% \\
3.3 \% \\
3.3 \%\end{array}$ \\
\hline
\end{tabular}

Table-III

Etiology of recurrent/persistent pneumonia $(n=30)$

\begin{tabular}{lcc}
\hline Etiology & Number & Percentage \\
\hline GERD & 2 & $6.6 \%$ \\
FB aspiration & 2 & $6.6 \%$ \\
Cystic fibrosis & 4 & $13.3 \%$ \\
Immune deficiency & 3 & $10 \%$ \\
Bronchial asthma & 3 & $10 \%$ \\
Pulmonary TB & 7 & $23.3 \%$ \\
Congenital Lung Agenesis & 1 & $3.3 \%$ \\
Bronchiectasis & 2 & $6.6 \%$ \\
Congenital heart disease & 5 & $16.6 \%$ \\
Unknown cause & 1 & $3.3 \%$ \\
\hline
\end{tabular}

\section{Discussion:}

There are several studies of recurrent and/or persistent pneumonia worldwide. Most of the studies highlighted the underlying causes ${ }^{4-8,11-14}$. In this study $80 \%$ patients were recurrent and $20 \%$ patients were persistent pneumonia and the most frequent underlying cause was pulmonary TB, followed by congenital heart disease, cystic fibrosis, immune deficiency disorders, bronchiectasis, and congenital lung agenesis. The current results and a review of the literature revealed the causes of recurrent/ persistent pneumonia in children from different geographical areas are similar but of variable prevalence. Children had almost similar onset of symptoms at an early age. Saad et al. found the most common cause was aspiration syndrome $(17.7 \%)$ followed by pulmonary TB (14.0\%) and congenital heart disease (11.5\%). Owayed et al found the most common cause was aspiration syndrome (48\%), immune disorders $(10 \%)$, congenital heart disease $(9 \%)$ and asthma (9\%).

In our study, pulmonary TB was diagnosed in 7 (23.3\%) patients; 2 with recurrent pneumonia and 5 with persistent pneumonia. Mantoux test was positive in all cases. Acid fast bacilli were found in $\mathrm{ZN}$ staining of the gastric lavage with 4 patients. Gene X-pert was positive for MTB in 5 patients with sensitive to rifampicin. The remaining 2 patients were diagnosed on the basis of strong clinical and radiographic findings along with positive contact history with known adult cases of tuberculosis. These patients did not respond to usual antibiotics and had clinical improvement on anti-tuberculous therapy.

The current study showed that 4 cases had CF, diagnosed by positive sweat chloride test. Patients were diagnosed depending on the history of pneumonia, failure to thrive, consanguinity of parents and physical findings and positive sweat chloride test (Conventional bag method). Two patients had pseudomonas aeruginosa in the culture of the secretions from respiratory tract and patients responded well with anti-pseudomonal treatment which is also highly suggestive of cystic fibrosis ${ }^{15-17}$. Although the prevalence of CF is rare in our country, we diagnosed 3 cases presented with respiratory symptoms alone and 1 case with respiratory symptoms associated with malabsorption.

Congenital heart disease is one of the important causes for recurrent/persistent pneumonia in 
children. Congenital acyanotic heart diseases with left-to-right shunts cause increase in pulmonary blood flow, cardiac workload and increase the susceptibility to respiratory infections ${ }^{18}$. Cyanotic congenital heart diseases with increased pulmonary blood flow also cause recurrent and persistent pneumonia. A review of the literature revealed that congenital heart disease accounts for $1.2-25.4 \%$ of cases $4,6,7,9,11,12,19,20$.Our current study finds it $16.6 \%$ which is consistent with previous results. Among the shunt lesions that present in infancy, ventricular septal defect is the most common. Then Atrial septal defect, Patent ductus arteriosus, Transposition of great arteries(TGA) occurs.

Immune deficiency disorders were found in 3 patients $(10 \%)$ which is consistent with previous studies that demonstrated immune deficiency disorders in 7.7$17.75 \%$ of cases $^{4,10,12,19,20}$. All of our cases were hypogammaglobulinemia and had recurrent pneumonia. Children with immune deficiency present with recurrent and/or severe infections that may be caused by unusual organisms or that involve multiple sites in addition to the lungs ${ }^{22,23}$. Immunoglobulin replacement therapy reduced the frequency and severity of infections in hypogammaglobulinemia, although long-term pulmonary complications may occur. ${ }^{21}$

Bronchial asthma is a common respiratory illness and also considered as a cause of recurrent and persistent pneumonia usually in older children ${ }^{11,24}$. Often pneumonia may be the initial symptom event without classical presentation of bronchial asthma. We found in 3 patients $(10 \%)$ who were diagnosed clinically and functionally. Our finding is consistent with the findings of previous studies $6,11,12,14$ who found bronchial asthma in $15 \%-69 \%$ of cases.

Aspiration syndrome accounted for $13.3 \%$ of our patients; 2 of them were secondary to GERD, and 2 had FB aspiration. GERD is the important cause of recurrent aspiration. Pharyngeal incoordination is also an important cause, particularly in children with cerebral palsy or other neuromuscular dysfunction. Patients with FB aspiration were advised for bronchoscopic removal of foreign body in chest disease hospital. Most of the studies found aspiration syndrome as the most frequent cause of recurrent/ persistent pneumonia ${ }^{4,9,19,20}$.

We found 2 children (6.6\%) who had bronchiectasis, no cause of which could not be identified. One patient had history of measles though it was difficult to diagnose post measles bronchiectasis. Patients CT scan report was suggestive of bronchiectasis and patients were discharged with supportive treatment and advised for follow up.

We found one patient (3.3\%) with congenital agenesis of right lung which was diagnosed by CT scan of chest. Patient had evidence of persistent pneumonia. Previous studies reported congenital anomalies of the respiratory tract in $3.7-8.5 \%$ of cases. $4,19,20$.

This retrospective study had some limitations. Our data were limited to children who were evaluated in one unit; the most encountered known etiologies were pulmonary TB, and congenital heart disease. Tuberculosis is a communicable disease. Proper health care measures should be taken to prevent transmission of TB among pediatric age group. Congenital heart disease should also be looked for carefully for the management of recurrent/persistent pneumonia.

\section{References:}

1. McIntosh K. Community-acquired pneumonia in children. N Eng J Med. 2002 Feb 7;346(6): 429-37.

2. Garenne M, Ronsmans C, Campbell H. The magnitude of mortality from acute respiratory infections in children under 5 years in developing countries. World Health Stat Q. 1992;45:18091.

3. Wald E. Recurrent and non-resolving pneumonia in children.Semin Respir Infect 1993; 8: 46-58.

4. Owayed AF, Campbell DM, Wang EEL. Underlying causes of recurrent pneumonia in children. Arch Pediatr Adolesc Med 2000; 154: 190-4.

5. Lodha R, Puranik M, Natchu UC, Kabra SK. Recurrent pneumonia in children: clinical profile and underlying causes.Acta Paediatr 2002; 91(11): 1170-3.

6. Çiftçi E, Günep M, Köksal Y, Ýnce E, Doðru Ü. Underlying causes of recurrent pneumonia in Turkish children in a university hospital. J Trop Pediatr 2003; 49(4): 212-5.

7. Cabezuelo Huerta G, Vidal Micó S, Abeledo Gómez A, Frontera Izquierdo P. (Underlying causes of recurrent pneumonia). An Pediatr (Barc) 2005; 63(5): 409-12. 
8. Lodha R, Kabra SK. Recurrent/persistent pneumonia. Indian Pediatr. 2000;37:1085-92.

9. Kumar M, Biswal N, Bhuvaneswari V, Srinivasan S. Persistent Pneumonia: Underlying Cause and Outcome. Indian J Pediatr. 2009;76:12236.

10. Adam KAR. Persistent or recurrent pneumonia in Saudi children seen at King Khalid University Hospital, Riyadh: clinical profile and some predisposing factors. Ann Trop Paediatr 1991; 11:129-35

11. Eigen H, Laughlin JJ, Homrighausen J. Recurrent pneumonia in children and its relationship to bronchial hyperreactivity .Pediatrics 1982; 70: 698-704

12. Ozdemir O, Sari S, Bakirtas A, Zorlu P, Ertan U. Underlying diseases of recurrent pneumonia in Turkish children. Turk J Med Sci. 2010;40(1): 25-30.

13. Paulina B, Olate P, Enrique L, Eliana Munoz B, Linus Holmgren $P$, Ignacio Sànchez D. Clinical characteristics, epidemiology and risk factors of recurrent pneumonia in children. Rev Chil Pediatr. 2004;75:434-40.

14. Lodha R, Puranik M, Chandra U, Natchu M, Kabra SK. Persistent pneumonia in children. Indian Pediatr. 2003;40:967-70. http:// www.indianpediatrics.net/oct2003/oct-967970.htm.

15. Patria MF, Esposito S. Recurrent Lower Respiratory Tract Infections in Children: A
Practical Approach to Diagnosis. Ped Res Rev. 2013;14:53-60.

16. Couriel J. Assessment of the child with recurrent chest infections. Br Med Bull. 2002;61:115-32.

17. Panitch HB. Evaluation of Recurrent Pneumonia. Ped Infec Dis J. 2005;24:265-6.

18. Vaughan D, Katkin JP. Chronic and recurrent pneumonias in children. Semin Respir Infect. 2002;17:72-84.

19. Çelebi S, Hacimustafaoglu M, Albayrak Y, Bulur N. Recurrent Pneumonia in Children. Turk Thor J. 2010;11:2, 56-9.

20. Hoving MP, Brand PL. Causes of recurrent pneumonia in children in a general hospital. J Paediatr Child Health. 2013.

21. Patria MF, Esposito S. Recurrent Lower Respiratory Tract Infections in Children: A Practical Approach to Diagnosis. Ped Res Rev. 2013;14:53-60.

22. Haeney M. The detection and management of primary immunodeficiency. In: David TJ, editor. Recent Advances in Paediatrics. Vol. 9. Edinburgh: Churchill Livingstone; 1990. pp. 2140.

23. Couriel J. Assessment of the child with recurrent chest infections. Br Med Bull. 2002;61:115-32.

24. Kjellman B. Bronchial asthma and recurrent pneumonia in children: clinical evaluation of fourteen children. Acta Paediatr Scand 1967; 56: 651-9. 